\title{
Secondary Syphilis During Pregnancy: The Importance of Screening and Clinical Management
}

\author{
Indah Purnamasari, Jusuf Barakbah, Sunarko Martodihardjo, Dwi Murtiastutik, \\ Maylita Sari, Astindari, Septiana Widyantari, Afif Nurul Hidayati \\ Department of Dermatology and Venereology, Faculty of Medicine, Universitas Airlangga, Dr. \\ Soetomo General Academic Hospital, Surabaya, Indonesia.
}

\begin{abstract}
Background: Syphilis is one of the most serious sexually transmitted diseases worldwide, and has tremendous consequences for the mother and her developing fetus if left untreated. The burden of morbidity and mortality due to congenital syphilis is high. Purpose: Screening and prompt to know the importance of treatment of syphilis during pregnancy. Case: A 32-year-old multigravida in 5 months of pregnancy presented with multiple raised lesions over her labia. It was accompanied by fluor and smelly fishy odor. There was no history of genital ulcers in either spouse and no history of sexual promiscuity. History of antenatal care in public health showed non-reactive status of HIV, syphilis and hepatitis B. Clinical examination revealed multiple flat, moist warts over her labia mayora and minora, and multiple roseola syphilitica on the plantar pedis sinistra. Darkfield microscopic examination presence spirochete, venereal disease research laboratory (VDRL) titer was 1:16 and $T$. pallidum particle agglutination assay (TPHA) titer was 1:2560. Obstetric ultrasonography examination was suggestive no mayor congenital abnormalities. Both of serology VDRL and TPHA were non-reactive in her husband. Significant of lesion improvement and decrease a fourfold titer serologic in VDRL (1:4) and TPHA (1:320) as follow-up 3 months after being treated with single intra-muscular injections of benzathine penicillin 2.4 million units. Discussion: Coordinated prenatal care and treatment are vital. It's implemented before the fourth month of pregnancy to reduce vertical transmission and all associated side effects of congenital syphilis. Penicillin is highly efficacious in maternal syphilis and prevention of congenital syphilis. Conclusion: Universal screening and adequate pregnancy care must be a priority.
\end{abstract}

Keywords: pregnancy, secondary syphilis, screening, management, sexually-transmitted disease.

Corespondence address: Afif Nurul Hidayati, Department of Dermatology and Venereology, Faculty of Medicine, Universitas Airlangga, Dr. Soetomo General Hospital, Surabaya, Indonesia, Jl. Mayjen Prof Dr. Moestopo No. 6-8 Surabaya 60131, Indonesia. Telepon: +62315501609, e-mail: afif_nurulhidayati@yahoo.com.

\section{BACKGROUND}

Syphilis is a sexually transmitted disease (STD) caused by the bacterium Treponema pallidum, subspecies Pallidum. It's a major disease worldwide, and it potentially causes life-long health problems with the potential manifestation of multiple patterns of skin and visceral disease. Untreated syphilis in pregnancy leads to adverse outcomes among more than half of women with active diseases, including early fetal loss, stillbirth, preterm birth, low birth weight, neonatal and infant death, and congenital disease among newborn babies. $^{1,2}$

The World Health Organization (WHO) estimates that 1.3 million pregnant women annually have active syphilis infections. In 2015, the Centres for Disease Control (CDC) released a report warning of a $38 \%$ increase in reported cases of congenital syphilis between 2012 and 2014. . $^{3,4}$

Syphilis antenatal screening and treatment for pregnant infected women prevent mother-to-child transmission (MTCT) and aligns with the Sustainable Development Goal (SDG) targets of ending preventable deaths of newborns and children under 5 years of age, ensuring universal access to sexual and reproductive healthcare services, and achieving Universal Health Coverage (UHC). ${ }^{5}$

Penicillin is still the drug of choice of syphilis in pregnancy. There are no recommended alternate regimens for women with penicillin allergy and desensitization to penicillin is recommended. If syphilis is diagnosed during pregnancy, fetal ultrasonography should be performed to evaluate the presence of congenital syphilis. ${ }^{3,5}$

This report aims to describe the importance of syphilis screening and prompt treatment for pregnant women, thus knowing the optimal timing of antenatal interventions to prevent mother-to-child transmission of syphilis and its associated adverse outcomes.

\section{CASE REPORT}

A 32-year-old multigravida in 5 months of pregnancy presented multiple raised lesions over her labia of 5 days duration. She also reported complaints of minimal vaginal discharge, sometimes itchy, smelly 
fishy odor, and greyish color. Previous similar symptoms were denied. There was no history of genital ulcers in either spouse and no history of sexual promiscuity in the patient. Her last sexual contact with her husband was 2 weeks before the complain appeared.
Physical examination over the labia mayora et minora showed that there were multiple flat-topped papules well-demarcated, moist, whitish colored with the greyish white vaginal discharge homogenous, stick to the vaginal wall. In plantar pedis sinistra, there were multiple erythematous maculopapular, annular, welldemarcated covered with thin scale.

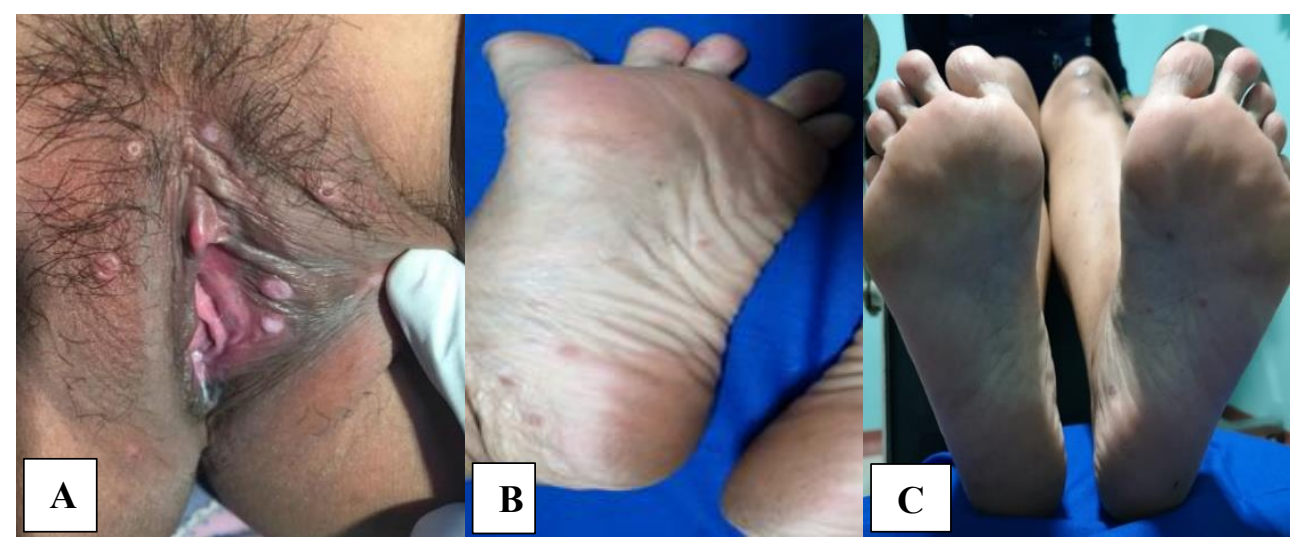

Figure 1. A. Multiple flat-topped papules, well-demarcated, moist, whitish over labia mayora, labia minora. B and C. Multiple erythematous maculopapular, annular, well-demarcated covered with a thin scale on plantar pedis sinistra

Darkfield microscopic examination showed a presence of spirochete, the characteristic of $T$. pallidum. Gram staining, and wet preparations from urethral and vaginal swab showed clue cell, 20\% of all epithelium with vaginal $\mathrm{pH} 7$ and positive sniff test, fishy odor when adding one drop of 10-20\% potassium hydroxide of vaginal fluor. Serology test revealed a reactive VDRL and TPHA in titer 1:16 and $1: 2560$. Obstetric ultrasonography examination result showed a single fetus, alive, with a positive heart rate, suitable to 20-21 weeks of gestation, and not suggestive of mayor congenital abnormalities. No abnormality was noted in her husband and both VDRL and TPHA non-reactive. HIV test results were negative in both. Based on history, physical and supporting examination, the diagnosis was secondary syphilis (condyloma lata and roseola syphilitica) with bacterial vaginosis and 20-21 weeks of pregnancy single live intrauterine fetus.

Significant improvement on all lesions was observed after 1 month of 2.4 million IU of Benzathine penicillin $\mathrm{G}$ intramuscular injection, a single dose therapy. Further serologic examinations following up 3 months after the initial therapy showed a fourfold titer decrease of VDRL test (1:4) and TPHA test (1:320). The patient also received metronidazole intravaginal every night for 5 days.

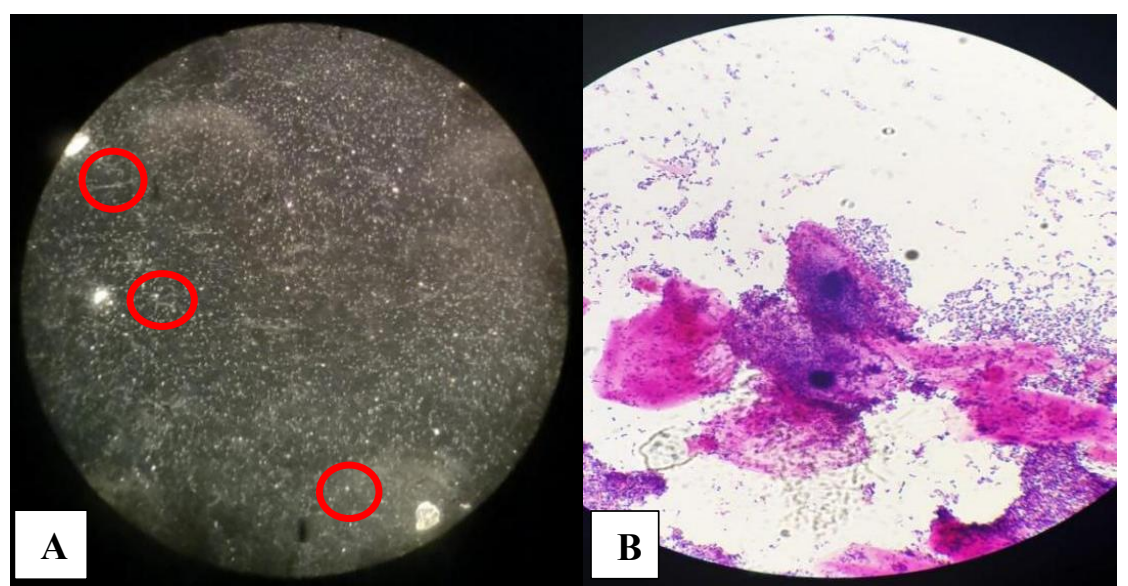

Figure 2. Microscopic examination. A. Darkfield examination showed the presence of organism with the characteristic morphology and motility of T. pallidum. B. Clue cell, $20 \%$ of all epithelium presented in Gram staining. 


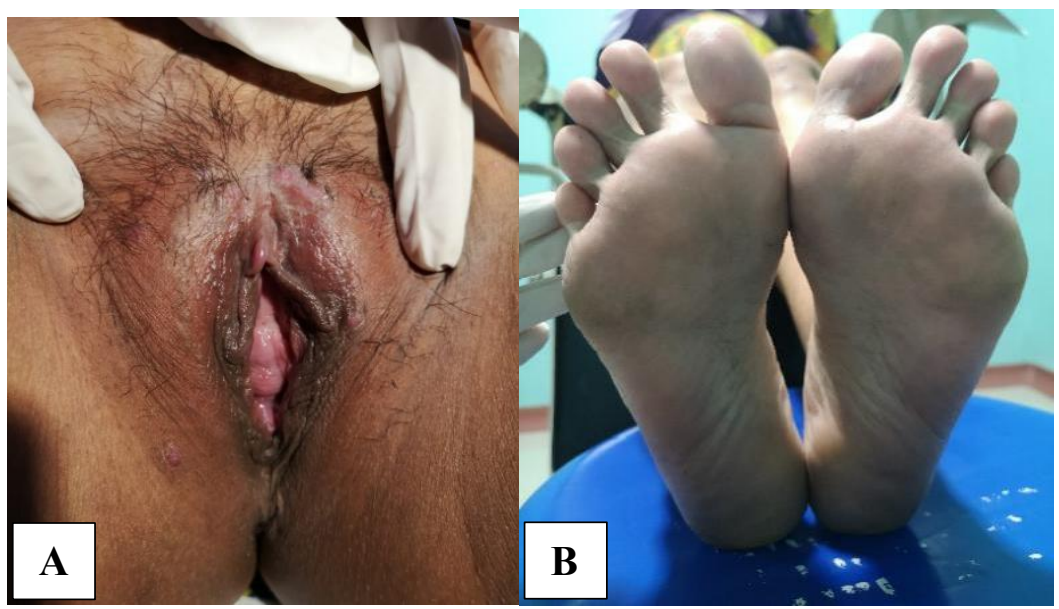

Figure 3. Improvement after 1 month treatment there is no condyloma lata and roseola Syphilitica.

\section{DISCUSSION}

Syphilis is a sexually transmitted disease that is known to have varied presentations; therefore, it is known as the 'Great Imitator'. The syphilis-affected pregnancies resulted in an estimated 350,000 adverse birth outcomes due to congenital syphilis in 2012. More than 1.4 million pregnant women have been infected with HIV, and mother-to-child transmission (MTCT) of HIV was estimated to have resulted in over 150,000 infant cases in 2015.,

The demographic profile of women who deliver syphilitic babies represents women with other sexually transmitted diseases (STDs) as well as those who fail to receive adequate prenatal care. ${ }^{6}$

Classically, syphilis infection is divided into 4 distinct clinical phases. They are primary, secondary, latent, and tertiary stages. Syphilis is spread through sexual contact and the spirochete penetrates mucous membranes or abrasions in the skin to disseminate systemically before the clinical disease appears. The incubation period ranges from 21 days to 6 months. A primary lesion, or painless chancre, appears at the site of inoculation. Without treatment, spontaneous resolution of the chancre will occur within 4-6 weeks on average. Secondary syphilis occurs 3 to 12 weeks following untreated primary disease and consists of systemic and localized or diffuse mucocutaneous lesions (roseola syphilitica), often with generalized lymphadenopathy. Condyloma lata is characteristically seen in secondary syphilis. They are essentially vegetative hypertrophy of the epidermis, confluent, pale-colored broad, and flattened papules occurring in warm moist areas. They begin as raised, slightly mammillated, or smooth papules with moist eroded surfaces and are more common in women than men due to moisture and friction in female genitalia, favoring their development. In women, the papules occur more commonly around the anus, the vulva, and the cervix. Thereafter, the patient enters the latent phase of syphilis, characterized by the absence of clinical symptoms but positive serologic tests. Syphilis can develop in up to one-third of untreated patients. This includes benign granulomatous lesions of the skin, mucous membranes, skeleton, and involvement of the aorta (cardiovascular syphilis). The majority of deaths from tertiary syphilis are the result of cardiovascular involvement. ${ }^{1,2}$

Maternal syphilis can be suspected based on clinical findings and confirmed by direct identification of treponemes in clinical specimens as well as by positive serologic findings or can be accidentally diagnosed through screening serological tests. Darkfield microscopy examination is the most specific direct technique for diagnosing syphilis when an active chancre or condyloma latum is present. A positive result means the presence of organisms with the characteristic morphology and motility of T. pallidum. Darkfield microscopic examination often shows positive results before serologic tests become reactive. Its sensitivity is approximated within $74 \%$ to $79 \%$, but declines as minutes elapse, as dead treponemes cannot exhibit the motility required for diagnosis..$^{1,7}$

In this case, the patient was reported with multiple flat-topped papules over her vagina, moist and whitish, known as condyloma lata as well as reported with multiple erythematous maculopapular, annular, welldemarcated covered with a thin scale on the left soles of the foot known as roseola syphilitica. The darkfield microscopic examination of condyloma lata over labia mayora showed an presence of spirochete, the organism with the characteristic morphology and motility of T. pallidum. Serological testing is the most common method for syphilis screening, diagnosis, and follow-up of treatment. The T. pallidum particle agglutination assay (TPHA) can be used in combination with Venereal Disease Research Laboratory (VDLR) or RPR to maximize the detection of primary infection on screening. Secondary syphilis 
is always characterized by a reactive VDRL, with a titer greater than $1 / 16 .{ }^{1,7}$ In this case, serology test for syphilis revealed a reactive VDRL and TPHA in titer $1: 16$ and 1:2560. One month follow-up after the therapy of Benzathine penicillin $G$ injection showed that the VDLR remains the same at 1:16 and TPHA 1: 2560. After three months follow-up, the VDRL result decreased to $1: 4$ and TPHA to $1: 320$. It indicated a positive response to the treatment.

The international guidelines and WHO recommend serological screening syphilis tests for all pregnant women in the first antenatal visit and repeated in 32-36 weeks, if the woman is at risk for syphilis. Moreover, any woman who delivers a stillborn infant after 20 weeks gestation should be tested for syphilis. Most importantly, this patient was seen in 20-21 weeks gestation and treatment commenced immediately, thereby minimizing the risk of vertical transmission to 70-100 percent. $^{2}$ Empirical study on how to enhance early attendance frequency in antenatal care is essential to ensure that the first prenatal visit happens early enough in pregnancy for treatments to be most effective. If syphilis screening and treatment are delayed during pregnancy, congenital syphilis is more likely. ${ }^{8}$

Benzathine penicillin $G$ is highly efficacious during pregnancy, and it remains the only recommended treatment for maternal syphilis and the prevention of congenital syphilis. Studies have found the efficacy to be $99.7 \%$ for eradicating maternal disease during pregnancy and $98.2 \%$ for preventing congenital syphilis across all stages of syphilis. ${ }^{9}$ The management of this case was 2.4 million IU of Benzathine penicillin $G$ injection, a single intramuscular dose, with a skin test first.

Early and appropriate maternal treatment during pregnancy has repeatedly been demonstrated to result in the lowest incidence of congenital syphilis and poor pregnancy outcomes. If any BPG dosage is missed, or if there is a gap of more than 10 days between doses, the full regimen should be repeated. In the same way, if a gravida is treated with anything other than penicillin, adequate therapy cannot be guaranteed. ${ }^{8,10}$

This has recently been called into doubt due to a nationwide scarcity of BPG. Although there have been no randomized controlled studies comparing 1 dose with 2 doses for early syphilis during pregnancy, there are theoretical pharmacologic advantages to justify extra doses. ${ }^{11}$

The CDC particularly stresses the importance of proper diagnosis and management of sexual partners of the patient with syphilis. It has been estimated that up to $60 \%$ of sexual contacts of patients with syphilis may be infected. Sexual partner management is critical to prevent reinfection of the pregnant women. ${ }^{12}$

The triple elimination program of HIV, syphilis, and Hepatitis B transmission is carried out to prevent transmission especially in mothers infected with HIV, syphilis, and/or hepatitis B. The indicators used are new HIV infections, syphilis, and/or hepatitis B in children that are less than or equal to $50 / 100,000$ live births. The triple elimination is carried out through health promotion activities, health surveillance, early detection, and/or case handling. Early detection of triple elimination through blood tests at least once during pregnancy during antenatal care (ANC). The WHO recommendation is before 20 weeks gestation, and the initial syphilis screening should be done in the first trimester and repeated on 28 weeks and during labor. Coordinated prenatal care and treatment are vital. The goal of therapy does not only address the immediate syndrome, but also to prevent transmission to sexual partners, and progression to tertiary syphilis. ${ }^{13}$

Quality improvement and monitoring efforts at many levels are required to attain and sustain these objectives. The procurement of commodities like HIV and syphilis diagnostic tests and medicines is a key component of keeping triple elimination services running. The requirement for reliable supply of benzathine penicillin for the treatment of maternal infections is particularly pressing. For syphilis, support for laboratory quality assurance is required to ensure that diagnostic tests are of adequate quality and that inter-pretation criteria are fulfilled. For point-of-care syphilis testing, quality assurance is also required. The close monitoring of syphilis-affected babies necessitates the use of sophisticated tracking devices. ${ }^{14,15}$

Syphilis in pregnancy causes adverse birth outcomes due to congenital syphilis. It's could be recognized, tested, and timely treatment during pregnancy. Early screening and diagnosis of syphilis should be performed in pregnancy or a suspected individual. The best choice of treatment during pregnancy is penicillin.

\section{REFERENCES}

1. Tuddenham SA, Zenilman JM. Syphilis. In: Goldsmith LA, Katz SI, Gilchrest BA, Paller AS, Leffell DJ, Wolff K, editors. Fitzpatrick's Dermatology in General Medicine. $9^{\text {th }}$ edition. New York : Mc Graw Hill. 2019. p. 3145-68.

2. World Health Organization. Guidelines on syphilis screening and treatment for pregnant women. Geneva: WHO; 2017. 
3. Centres for Disease Control and Prevention. Incidence, prevalence, and cost of Sexually Transmitted Infections in the United States. CDC Fact Sheet. 2013. p.1-4.

4. Schmid G. Economic and programmatic aspects of congenital syphilis prevention. Bulletin of the World Health Organization 2004; 82(6): p. $402-$ 9.

5. Taylor M, et al. Elimination of mother-to-child transmission of HIV and Syphilis (EMTCT): Process, progress, and program integration. PLoS Med 2017; 14(6): p. e1002329.

6. Genc M, Ledger WJ. Syphilis in pregnancy. Sex Transm Inf 2000; 76: p. 73-9.

7. Santis M, Luca C, Mappa I, Spagnuolo T, Licameli A, Straface G. Syphilis infection during pregnancy: fetal risks and clinical management. Hindawi Publishing Corporation Infectious Diseases in Obstetrics and Gynecology. 2012. p. 1-6.

8. Hawkes S, Matin N, Broutet N, Low N. Effectiveness of interventions to improve screening for syphilis in pregnancy: a systematic review and meta-analysis. Lancet Infect Dis; 11.p. 684-91.

9. Martha W.F, Paula A.R, Catherine S.P. Syphilis during pregnancy: a prevebtable threat to maternal fetal health. Am J of Obstet and Gynecol 2016; 216(4): p. 352-63.
10. Zhang Xh, Xu J, Chen DQ, Guo LF, Qiu LQ. Effectiveness of treatment to improve pregnancy outcomes among women with syphilis in Zhejiang Province, China. Sex Transm Infect 2016;0:1-5.

11. Walker GJ. Antibiotics for syphilis diagnosed during pregnancy. Cochrane Database Syst Rev. 2001;(3):CD001143

12. Centres for Disease Control and Prevention (CDC). Syphilis. Sexually Transmitted Disease Treatment Guideline, 2015. p. 34-50.

13. Republic of Indonesia Minister of Health Regulation. Regulation of the Minister of Health of the Republic of Indonesia No. 52, 2017 about Elimination of Transmission of Human Immunodeficiency Virus, Syphilis, and Hepatitis B from Mother to Child. Jakarta. Available at: http://hukor.kemkes.go.id

14. Taylor MM, Zhang X, Nurse-Findlay S, Hedman L, Kiarie J. Global penicillin need to prevent mother-to- child transmission of syphilis. Bull World Health Organ. 2016; 94(8):559-559A. https://doi.org/10.2471/ BLT.16.173310 PMID: 27516630

15. World Health Organization. Global Health Observatory. http://apps.who.int/gho/data/node.main. Accessed November 18, 2016. 